\title{
Evaluación de las propiedades psicométricas de reproducibilidad del Oral Health Impact Profile (OHIP-49) entre pacientes con enfermedad periodontal
}

\section{Psychometric reproducibility properties analysis of the Oral Health Impact Profile (OHIP-49) in patients with periodontal disease}

Avaliação das propriedades psicométricas de reprodutibilidade do Oral Health Impact Profile (OHIP-49) em pacientes com doença periodontal

\section{Renata De La Hoz Perafán ${ }^{1}$ Erica Tatiana Loaiza Echeverri² Iván Darío Arroyave Zuluaga ${ }^{3}$}

Recibido: 13 de agosto de 2019 Aprobado: 30 de noviembre de 2019 Publicado: 30 de diciembre de 2019

Cómo citar este artículo: Loaiza E, Arroyave I, De La Hoz RP. Evaluación de las propiedades psicométricas de reproducibilidad del Oral Health Impact Profile (OHIP-49) entre pacientes con enfermedad periodontal. Revista Nac. Odontol. (2019); 15(29), 1-27. doi: https://doi.org/10.16925/2357-4607.2019.02.12

Artículo de investigación. https://doi.org/10.16925/2357-4607.2019.02.12

1 Facultad de Ciencias de la Salud, Universidad del Magdalena, Santa Marta, Magdalena, Colombia. Escuela de Graduados, Universidad CES, Medellín, Colombia.

ORCID: https://orcid.org/0000-0002-4411-3029

Correo electrónico: rdelahoz@unimagdalena.edu.co

Facultad de Veterinaria, Universidad Pontificia Bolivariana, Medellín, Colombia.

Escuela de Egresados, Universidad CES, Medellín, Colombia.

ORCID: https://orcid.org/0000-0003-3589-6786

Facultad Nacional de Salud Pública, Universidad de Antioquia, Medellín,

Colombia. Escuela de Egresados, Universidad CES, Medellín, Colombia.

ORCID: https://orcid.org/0000-0001-9989-5833 


\section{Resumen}

Introducción: la medición de la calidad de vida relacionada con la salud oral es un indicador de la afectación negativa de la vida diaria de los pacientes por su condición oral. El OHIP-49 es un instrumento de uso extendido, los investigadores presentan el análisis de las propiedades psicométricas que describan el comportamiento del instrumento en la población de estudio. El objetivo es evaluar las propiedades de reproducibilidad del instrumento OHIP-49 entre pacientes con enfermedad periodontal que asisten a dos instituciones prestadoras de salud.

Métodos: con una muestra de 207 participantes, como lo sugiere la International Test Comission, se incluyeron por conveniencia 91 hombres y 116 mujeres; se evaluaron las propiedades de reproducibilidad: fiabilidad, consistencia interna, poder discriminante y validez de contenido.

Resultados: se obtuvo un alpha de Cronbach de 0,951 y altos porcentajes de éxito para la consistencia (87,5\%$100 \%)$; el poder discriminante tuvo aceptables porcentajes de éxito (16,6\%-68,5\%). Se decide revisar la estructura del instrumento y se obtuvo un modelo de siete factores. Según la condición clínica, los pacientes con periodontitis estadíos III y IV presentaron mayor afectación en las dimensiones de limitación funcional, masticación, deglución y estética al compararlos con los casos menos severos.

Conclusiones: se obtuvo un buen comportamiento del instrumento en esta población de la costa caribe colombiana, pero es válido considerar reducir dimensiones para mejorar los puntajes de reproducibilidad.

Palabras clave: estudios de validación, calidad de vida, periodontitis, epidemiología, estadística.

\section{Abstract}

Introduction: the oral health related quality of life measurement is considered a daily life negative effect indicator due to patients' oral condition. The Oral Health Impact Profile (OHIP-49) is an extended-use instrument, the readers should to expect the researchers to present the psychometric properties analysis, which describe the instrument behavior in the study population. The purpose is to evaluate the OHIP-49 reproducibility properties when is applied in patients with periodontal disease, who attend two dental healthcare institutions.

Methods: we included 207 participants how it is suggested from the International Test Commission for similar purposes. We included 91 men and 116 women for convenience; reproducibility properties were evaluated: reliability, internal consistency, item discriminant validity, and content validity.

Results: Cronbach's alpha of 0.951 was obtained and high success percentages for internal consistency ( $87.5 \%$ $-100 \%)$; the item discriminant validity not achieved acceptable success percentages $(16,6 \%-68,5 \%)$. We decided to review the instrument content structure, with which a seven-factor model was obtained. According to the clinical situation, when we compared them with the less severe cases, patients with periodontitis stage III and IV presented worst quality of life represented in the dimensions of functional limitation, chewing, swallowing and aesthetics.

Conclusions: a good instrument performance was obtained for this Colombian Caribbean Coast population; but it is valid to consider reducing dimensions to improve reproducibility scores.

Keywords: validation studies, quality of life, periodontitis, epidemiology, statistics.

\section{Resumo}

Introdução: a mensuração da qualidade de vida relacionada à saúde bucal é um indicador da afetação negativa da vida diária dos pacientes devido à sua condição bucal. O OHIP-49 é um instrumento amplamente utilizado; os pesquisadores apresentam a análise das propriedades psicométricas que descrevem o comportamento do 
instrumento na população estudada. 0 objetivo é avaliar as propriedades de reprodutibilidade do instrumento OHIP-49 em pacientes com doença periodontal atendidos em dois profissionais de saúde.

Métodos: com uma amostra de 207 participantes, conforme sugerido pela Comissão Internacional de Testes, 91 homens e 116 mulheres foram incluídos por conveniência; Propriedades de reprodutibilidade: confiabilidade, consistência interna, poder discriminante e validade de conteúdo foram avaliadas.

Resultados: foi obtido um alfa de Cronbach de 0,951 e altas taxas de sucesso para consistência ( $87,5 \%-100 \%)$; o poder discriminador teve taxas de sucesso aceitáveis $(16,6 \%-68,5 \%)$. Decidiu-se revisar a estrutura do instrumento e um modelo de sete fatores foi obtido. De acordo com a condição clínica, os pacientes com periodontite estágios III e IV apresentaram maior comprometimento nas dimensões limitação funcional, mastigação, deglutição e estética quando comparados aos casos menos graves.

Conclusões: foi obtido um bom desempenho do instrumento nessa população da costa caribenha colombiana, mas é válido considerar a redução de dimensões para melhorar os escores de reprodutibilidade.

Palavras-chave: estudos de validação, qualidade de vida, periodontite, epidemiologia, estatística.

\section{Introducción}

La salud tiene un efecto directo sobre la calidad de vida. Desde hace más de una década la epidemiología ha usado escalas psicométricas para evaluar el impacto de la salud oral sobre la calidad de vida de los pacientes. Las escalas permiten resaltar la importancia de las patologías orales, fundamentando la necesidad de estrategias de prevención, tratamiento y evaluación del grado de afectación sobre la calidad de vida de los pacientes, que genera deficiencias a nivel físico y limitantes a nivel psicológico. Debido al componente multidimensional de la salud oral, incluyendo la calidad de vida, existen oportunidades para promover el empleo de acciones oportunas y efectivas como complemento para los criterios normativos que rigen la prestación de los servicios de salud odontológicos (1) (2).

Por sus manifestaciones clínicas, la enfermedad periodontal puede afectar la vida de los pacientes de forma negativa, en ocasiones, con un gran componente de deficiencias a nivel físico y limitante en lo psicosocial. La periodontitis es una enfermedad inflamatoria que destruye los tejidos que soportan los dientes, causando la pérdida de la dentadura y es una de las patología orales asociadas al detrimento de la percepción de la calidad de vida en los pacientes (3)-(5). Además, la periodontitis es considerada un evento negativo que puede asociarse a un estado depresivo que desencadena una inmunosupresión relativa. Estos factores, contribuyen al avance de la enfermedad debido a que el sistema inmunológico debería frenar la evolución de la misma (6). La periodontitis es considerada una de las enfermedades crónicas con más prevalencia en el mundo, pues se estima su presencia, en su condición más severa en el 11,2\% de la población mundial (7); mientras en Colombia, la periodontitis 
4 Evaluación de las propiedades psicométricas de reproducibilidad del Oral Health Impact Profile (OHIP-49) entre pacientes con enfermedad periodontal

alcanza a afectar al 61,8\% de la población, encontrándose su condición más severa en el $10,62 \%$ de los colombianos de acuerdo con los datos del último estudio nacional de salud oral (8).

Muchos de los estudios clínicos en pacientes con periodontitis, tratan de mostrar el impacto de la enfermedad sobre la calidad de vida relacionada con la salud oral, para lo cual suelen aplicarse distintas escalas específicas de medición en odontología, entre ellas el Oral Health Impact Profile (OHIP) (9) (10), escala que ha sido traducida a numerosos idiomas, entre ellos el inglés, portugués, español, mandarín, entre otros (11)-(19). El oHIP está asociado con el aumento del impacto negativo sobre la percepción de calidad de vida durante los últimos doce meses (10). La afectación negativa se debe a la destrucción avanzada del periodonto, que causa la pérdida de la integridad oral con manifestaciones clínicas como la inflamación y sangrado espontáneo de las encías, la halitosis favorecida por la acumulación de cálculos dentales, la ausencia de dientes o la movilidad dental progresiva, lo que genera alteraciones en la masticación y cambios en la posición dental que conllevan deficiencias no solo funcionales, sino también estéticas, que causan en el paciente mecanismos de defensa inconscientes, dentro de los que se encuentran limitar la interacción con otras personas o cambiar sus hábitos alimenticios para minimizar las molestias (2) (20)-(24).

Durante esta prueba piloto se realizó la evaluación de las propiedades psicométricas de reproducibilidad y validez de contenido de la escala OHIP-49, al aplicarlo en pacientes que asisten a dos clínicas odontológicas con distintos diagnósticos de enfermedad periodontal. Se espera que este artículo sea un referente de la relevancia clínica, además del impacto social logrado con los resultados obtenidos por el uso de este instrumento, y se favorezca su implementación con el rigor metodológico que conlleva su validación. El propósito de este artículo es evaluar las propiedades de reproducibilidad del OHIP-49 en pacientes con enfermedad periodontal que asisten a dos Instituciones Prestadoras de Salud en la ciudad de Santa Marta.

\section{Materiales y métodos}

Esta investigación tiene un enfoque cuantitativo, el tipo de estudio es descriptivo de corte transversal de evaluación psicométrica. Los participantes de la prueba piloto, como primera fase de este estudio, fueron 207 pacientes que asistieron a dos clínicas odontológicas en la ciudad de Santa Marta, una de las clínicas es Institución Prestadora de Salud (IPS) universitaria, mientras que la segunda IPS es privada y asisten pacientes afiliados al régimen contributivo y subsidiado del Sistema General de Seguridad Social en Salud (SGSSS). Lo anterior, con la finalidad de ampliar la 
variabilidad en las características sociodemográficas. La recolección de datos se realizó durante los meses de octubre del 2018 hasta marzo del año 2019, participaron tres encuestadores quienes recibieron entrenamiento en la Unidad de Atención Psicológica de la Universidad del Magdalena (UAP), con la finalidad de estandarizar la aplicación del instrumento. El criterio de selección de la muestra tuvo como base la Comisión Internacional de Test, que sugiere una muestra de al menos 200 participantes en casos de instrumentos ampliamente utilizados y donde la intención del autor es la identificación de ítems potencialmente sesgados, como se realizará en esta prueba piloto (25), esta cantidad permite la factorización de los datos. Dentro de los criterios de selección de los participantes se tuvo en cuenta que fueran pacientes mayores de 18 años, con al menos 18 dientes naturales en boca y que accedan a participar voluntariamente en el estudio. Se excluyeron los individuos con alteraciones mentales y/o cognitivas que pudieran afectar el diligenciamiento del instrumento o la obtención de datos. Esta investigación fue aprobada por el Comité Institucional de Ética en Investigación en Humanos de la Universidad CES (Acta 087 del 2015, Acta 123 del 2018).

El instrumento OHIP-49 consta de 7 dimensiones: limitación funcional (LF), dolor físico (DF), malestar psicológico (MP), incapacidad física (IF), incapacidad psicológica (IP), incapacidad social (IS), en desventaja (ED). Cada pregunta tiene 5 opciones de respuesta (tipo Likert) y a cada una se le asigna un puntaje: 0 "nunca", 1 "casi nunca", 2 "a veces", 3 "frecuentemente", 4 "siempre". El análisis del oHIP permite usar tres variables de resumen de las consecuencias funcionales y psicológicas de los problemas de salud bucal: 1) prevalencia: porcentaje de individuos que reportan uno o más ítems: "frecuentemente" o "siempre", 2) extensión: es el número de ítems reportados como "frecuentemente" o "siempre", 3) severidad: es la suma de todos los valores ordinales de las respuestas (26).

El valor obtenido del OHIP se calcula sumando todas las puntuaciones indicadas por los pacientes, la puntuación mínima es 0 , de manera que al multiplicar el valor máximo que cada impacto puede causar por el número de preguntas, se obtiene una puntuación máxima de 196 en el OHIP-49. Al valorar las categorías de cada dimensión, las puntuaciones obtenidas también pueden agruparse de la siguiente manera: puntaje de 0 a 2 componen el grupo con el menor impacto (nunca, casi nunca, a veces), aquellos con puntuaciones de 3 a 4 comprenden el grupo con los resultados de mayor impacto (cuando las respuestas eran: frecuentemente o siempre). En el oHIP las puntuaciones más altas indican mayores problemas de salud oral percibida por el paciente y las repercusiones de estos problemas sobre la calidad de vida relacionada con salud oral (2). El OHIP es ampliamente utilizado a nivel mundial, y previamente 
fue evaluada su validez de apariencia, contenido, constructo, criterio, y convergencia, logrando su validación en la población general. Por esa razón, la validez de apariencia y constructo no es requerida en este estudio, en que se evaluaron las propiedades de reproducibilidad del instrumento (27). Para esta investigación, se utilizó la versión chilena del instrumento (28).

\section{Análisis estadístico}

Se utilizó el paquete estadístico SPSS versión 25 y los resultados se contrastaron con los obtenidos en el paquete estadístico R. Las diferencias entre las variables demográficas y socioeconómicas entre los grupos que asistieron a las dos IPS fueron medidas usando la prueba de $X^{2}$ por ser de naturaleza cualitativa, todos los valores significativos (valor $p)$ corresponden pruebas de dos colas $(\alpha=0,05)$.

\section{Evaluación de las propiedades psicométricas de reproducibilidad}

Debido a que la población son pacientes de dos clínicas odontológicas no se consideró pertinente analizar la fiabilidad inter e intra-observador dado que, al recibir atención odontológica, se esperan cambios en la calidad de vida relacionada con la salud oral. Dentro de las propiedades psicométricas asociadas a la reproducibilidad que se evaluaron en este estudio se encuentran, la fiabilidad con la cual se determinó la proporción de la varianza total atribuible a diferencias verdaderas que existen entre los participantes (27), evaluando el grado en que un instrumento mide con precisión, sin error (29). El coeficiente de alfa de Cronbach es el recurso estadístico más utilizado para medir la fiabilidad de un instrumento y su valor mínimo aceptado es 0,7; aunque valores mayores a 0,9 puede ser indicio de duplicación. En general, siguiendo un perfil conservador, se prefiere valores de alfa de Cronbach entre 0,7 y 0,8 (30).

Con la consistencia interna se midió el grado de correlación y coherencia entre los ítems que conforman una dimensión, indicando que individualmente miden el concepto que tienen que medir y no otro concepto; esta propiedad se analizó utilizando el coeficiente de correlación de Spearman, al no seguir los datos una distribución normal, estos resultados también permiten establecer el porcentaje de éxito dentro de cada dimensión, se tendrán en cuenta los valores $\geq 0,4$ como valor de referencia. El poder discriminante, determina el grado de correlación que existe entre los ítems de una dimensión con respecto a las otras dimensiones a las cuales no pertenecen, esta 
propiedad puede ser medida con el rango del coeficiente de correlación de Spearman y, posteriormente, establecer el porcentaje de éxito para cada dimensión. Para este análisis se tendrán en cuenta valores $\leq 0,3$ como referencia de la menor correlación del ítem con las otras dimensiones (27).

\section{Análisis factorial exploratorio (AFE)}

Para la exploración de la estructura de la escala y la justificación de cada ítem en las dimensiones establecidas se utilizaron métodos de resumen y reducción de datos que, en este caso, fue el análisis factorial exploratorio (31) (32). Inicialmente, se verificaron el cumplimiento de los supuestos estadísticos del análisis factorial exploratorio (AFE) que son la identificación de datos extremos, la normalidad y la linealidad. La identificación de datos extremos se hizo a través del método de identificación de datos extremos en análisis univariado, utilizando el puntaje $Z$ que usa la media y la desviación estándar, en que el punto de corte de referencia son valores fuera del rango \pm 3 en muestras grandes, mientras que en muestras pequeñas de hasta 40 observaciones, el punto de referencia son valores >3,5 (33). Para la verificación de la normalidad se utilizó la prueba Shapiro-Francia; sin embargo, al considerarse demasiado sensible a pequeñas variaciones de la normalidad, la distribución fue medida de forma secundaria con la curtosis y la asimetría considerando que valores en el umbral $\pm 1,5$ son indicadores de variaciones leves y son adecuados para el AFE. La linealidad se evaluará examinando diagramas matriciales de dispersión; y la multinolinealidad al identificar correlaciones $>0,90$ (32).

Se hizo la medición de las características de la matriz de correlación, y el valor de su determinante $>0$, como referencia por permitir la manipulación matemática de la matriz en el AFE por tener asociación inversa. Esto se complementará con la prueba de esfericidad de Barttlet cuya hipótesis nula $(\mathrm{Ho})$ es que no existe correlación entre las variables $(p \leq 0,05)$. En ocasiones, esta prueba puede mostrar resultados significativos a pesar de no haber correlación y, por eso, se complementa con la prueba de Kaiser-Meyer-Olkin (KMO) como segundo indicador de la fuerza de correlación entre ítems y mostrando la adecuación de la muestra para la prueba con punto de corte $>0,70$ (31).

\section{Método de extracción}

Los métodos de ejes principales y de máxima verosimilitud han mostrado proporcionar los mejores resultados. Algunos autores consideran que a pesar de obtenerse 
resultados similares con el análisis de componentes principales no es del todo recomendable por ser un método de reducción de datos y no de una técnica de AFE. Al no seguir los datos una distribución normal, el método de extracción utilizado fue el de ejes principales (32). Inicialmente, se calculó la comunalidad como un estimado de la cantidad de varianza en cada ítem que es explicado por el factor extraído. Estos factores son extraídos sucesivamente y, por lo tanto, son ortogonales entre uno y otro, variables con comunalidades 0,50 carecen de explicación suficiente. El cálculo de los autovalores permite representar la cantidad de varianza total de los ítems que puede ser explicada por un factor dado. Se hizo el análisis de la matriz de correlación para analizar la significancia estadística de las correlaciones presentes $(p \leq 0,05)$. También se obtendrán los autovalores en análisis de ejes principales, representando la cantidad de varianza en todos los ítems, consolidando la varianza de la matriz cuyo valor se espera sea mayor a 0 , se extraen los factores con valores de referencia $<1$. Los valores de referencia serán para autovalores $\geq 1$; mientras que para la carga factorial valores $\geq 0,4$ que es un valor considerado importante al correlacionar cada ítem con el factor principal dado (34). Las soluciones obtenidas fueron contrastadas con las encontradas al aplicar el análisis de componentes principales, aunque este no es un método de análisis del factor, por su uso extendido, se decidió verificar la consistencia de sus datos con los hallados con el método seleccionado en esta investigación para analizar el factor que fue el método de ejes principales.

\section{Método de rotación}

Los resultados fueron sometidos al método de rotación ortogonal de Varimax, aunque también se utilizaron Quartimax y Equimax, aunque este último no presentó todas las matrices y tablas requeridas para el análisis utilizando el método de ejes principales. La rotación Varimax fue seleccionada porque, asumiendo que los factores son independientes, alcanza la máxima simplificación posible de las columnas de la matriz de correlaciones con fácil interpretación por sus valores cercanos a $-10+1$, indicando una clara asociación positiva o negativa entre la variable y el factor.

\section{Resultados}

Dentro de la muestra del estudio se encontraron similitudes entre los grupos en lo que concierne al sexo y al estado civil, dos variables asociadas con la percepción de la calidad de vida. Por el contrario, en relación con la edad hubo diferencias entre los grupos, con mayor frecuencia adultos entre los 18-44 y los de 45-65 años en la IPS 
universitaria, lo cual puede deberse a la diferencia en el tamaño de la muestra de cada IPS. Cabe resaltar, que las diferencias encontradas entre los grupos con respecto al nivel de escolaridad, estrato socioeconómico y ocupación, permite una adecuada variabilidad en el contexto social de los participantes lo que se refleja en la diversidad de las respuestas, motivo principal por el que se seleccionaron las dos IPS. Además, se hace evidente la baja frecuencia de periodontitis en la IPS privada (tabla 1).

Tabla 1. Descripción de los grupos participantes en el estudio.

\begin{tabular}{|c|c|c|c|c|c|}
\hline $\begin{array}{c}\text { Características } \\
\text { población }\end{array}$ & $\begin{array}{c}\text { Toda la } \\
\text { población } \\
n=207\end{array}$ & $\begin{array}{c}\text { IPS } \\
\text { Universitaria } \\
\mathrm{n}=139(66,2 \%)\end{array}$ & $\begin{array}{l}\text { IPS Privada } \\
n=68(32,4 \%)\end{array}$ & $\begin{array}{c}\text { Estadístico } \\
\mathrm{X}^{2}\end{array}$ & $\begin{array}{c}\text { Valor } \\
p\end{array}$ \\
\hline Edad & $41,6\left(\left.\mathrm{R}\right|^{*}=31-52\right)$ & $44(\mathrm{RI}=31-52)$ & $38,5(\mathrm{Rl}=25-27)$ & 79,909 & 0,001 \\
\hline 18-44 años & $113(53,8)$ & $70(50,4)$ & $43(63,2)$ & & \\
\hline 45-65 años & $89(42,4)$ & $65(46,8)$ & $24(35,3)$ & & \\
\hline$>65$ años & $8(3,8)$ & $4(2,9)$ & $1(1,5)$ & & \\
\hline Sexo & & & & 1,499 & $0,221^{\star *}$ \\
\hline Hombre & $91(44)$ & $57(41)$ & $34(50)$ & & \\
\hline Mujer & $116(56)$ & $82(59)$ & $34(50)$ & & \\
\hline Estado civil & & & & 10,128 & $0,072^{\star \star}$ \\
\hline Soltero & $74(35,7)$ & $50(36)$ & $24(35,3)$ & & \\
\hline Casado & $53(25,6)$ & $30(21,6)$ & $23(33,8)$ & & \\
\hline Viudo & $6(2,9)$ & $5(3,6)$ & $1(1,5)$ & & \\
\hline Separado & $9(4,3)$ & $9(6,5)$ & 0 & & \\
\hline Unión libre & $64(30,9)$ & $45(32,4)$ & $19(27,9)$ & & \\
\hline Divorciado & $1(0,5)$ & 0 & $1(1,5)$ & & \\
\hline Nivel de escolaridad & & & & 42,330 & 0,001 \\
\hline Sin educación & $16(7,7)$ & $16(11,5)$ & 0 & & \\
\hline Primaria & $58(28)$ & $48(34,5)$ & $10(14,7)$ & & \\
\hline Secundaria & $66(31,9)$ & $49(35,3)$ & $17(25)$ & & \\
\hline Técnico & $33(15,9)$ & $13(9,4)$ & $20(29,4)$ & & \\
\hline Universitario & $31(15)$ & $13(9,4)$ & $18(26,5)$ & & \\
\hline Posgrado & $3(1,4)$ & 0 & $3(4,4)$ & & \\
\hline Estrato socioeconómico & & & & 58,592 & 0,001 \\
\hline 0 & $4(2)$ & $3(2,2)$ & $1(1,5)$ & & \\
\hline 1 & $79(38,5)$ & $73(52,2)$ & $7(10,3)$ & & \\
\hline 2 & $50(24,4)$ & $38(27,3)$ & $13(19,1)$ & & \\
\hline 3 & $50(24,4)$ & $19(13,7)$ & $31(45,6)$ & & \\
\hline 4 & $15(7,1)$ & $4(2,9)$ & $11(16,2)$ & & \\
\hline 5 & $5(2,4)$ & $2(1,4)$ & $3(4,4)$ & & \\
\hline 6 & $2(1)$ & 0 & $2(2,9)$ & & \\
\hline
\end{tabular}


Evaluación de las propiedades psicométricas de reproducibilidad del Oral Health Impact Profile (OHIP-49) entre pacientes con enfermedad periodontal

(viene)

\begin{tabular}{|c|c|c|c|c|c|}
\hline $\begin{array}{c}\text { Características } \\
\text { población }\end{array}$ & $\begin{array}{c}\text { Toda la } \\
\text { población } \\
n=207\end{array}$ & $\begin{array}{c}\text { IPS } \\
\text { Universitaria } \\
\mathrm{n}=139(66,2 \%)\end{array}$ & $\begin{array}{l}\text { IPS Privada } \\
n=68(32,4 \%)\end{array}$ & $\begin{array}{c}\text { Estadístico } \\
\mathrm{X}^{2}\end{array}$ & $\begin{array}{l}\text { Valor } \\
p\end{array}$ \\
\hline Ocupación & & & & 28,355 & 0,001 \\
\hline Desempleado & $30(14,5)$ & $26(18,7)$ & $4(5,9)$ & & \\
\hline Empleado & $52(25,1)$ & $23(16,5)$ & $29(42,6)$ & & \\
\hline Hogar & $42(20,3)$ & $36(25,9)$ & $6(8,8)$ & & \\
\hline Independiente & $61(29,5)$ & $40(28,8)$ & $21(30,9)$ & & \\
\hline Estudiante & $20(9,7)$ & $14(10,1)$ & $6(8,8)$ & & \\
\hline Pensionado & $2(1)$ & 0 & $2(2,9)$ & & \\
\hline Enf. periodontal & & & & 53,331 & 0,001 \\
\hline Gingivitis & $115(54,8)$ & $53(38,1)$ & $62(91,2)$ & & \\
\hline Periodontitis estadío I & $35(16,7)$ & $34(24,5)$ & $1(1,5)$ & & \\
\hline $\begin{array}{l}\text { Periodontitis estadío } \\
\text { II }\end{array}$ & $31(14,8)$ & $30(21,6)$ & $1(1,5)$ & & \\
\hline $\begin{array}{l}\text { Periodontitis estadío } \\
\text { III y IV }\end{array}$ & $26(12,4)$ & $22(15,8)$ & $4(5,9)$ & & \\
\hline
\end{tabular}

${ }^{\star}$ Rango intercuartílico. ${ }^{\star \star}$ Grupos estadísticamente similares.

Fuente: elaboración propia.

Con respecto a las propiedades psicométricas de reproducibilidad, cada dimensión presentó excelente fiabilidad; sin embargo, el $\alpha$ de Cronbach global de 0,95 permite considerar la posibilidad de duplicidad, lo cual se ve apoyado por los bajos porcentajes de éxito presentados por las dimensiones en la columna del poder discriminante (tabla 2). Debido a esto, se decidió explorar la estructura factorial del instrumento implementando el AFE.

Tabla 2. Resultados análisis de reproducibilidad en la escala de calidad de vida relacionada con la salud oral en los pacientes que asisten a dos IPS en la ciudad de Santa Marta.

\begin{tabular}{|c|c|c|c|c|c|c|}
\hline \multirow[b]{2}{*}{ Dimensión } & \multirow[b]{2}{*}{$\begin{array}{l}\text { Número de } \\
\text { ítems por } \\
\text { dimensión }\end{array}$} & \multirow[b]{2}{*}{$\begin{array}{l}\text { Alfa de } \\
\text { Cronbach }\end{array}$} & \multicolumn{2}{|c|}{ Consistencia interna } & \multicolumn{2}{|c|}{ Poder discriminante } \\
\hline & & & $\begin{array}{c}\text { Punto- } \\
\text { Dimensión } \\
\text { Rango Rho }\end{array}$ & $\begin{array}{c}\% \\
\text { Éxito }\end{array}$ & $\begin{array}{l}\text { Punto-otra } \\
\text { dimensión } \\
\text { Rango Rho }\end{array}$ & $\begin{array}{c}\% \\
\text { Exxito }\end{array}$ \\
\hline Limitación funcional & 9 & 0,760 & $0,30-0,72$ & 88,8 & $0,04-0,66$ & 68,5 \\
\hline Dolor físico & 9 & 0,805 & $0,16-0,75$ & 88,8 & $0,12-0,48$ & 64,8 \\
\hline Malestar psicológico & 5 & 0,744 & $0,42-0,82$ & 100 & $0,00-0,66$ & 25 \\
\hline Incapacidad física & 9 & 0,830 & $0,23-0,74$ & 87,5 & $0,10-0$ & 27,7 \\
\hline
\end{tabular}




\begin{tabular}{|c|c|c|c|c|c|c|}
\hline \multirow[b]{2}{*}{ Dimensión } & \multirow[b]{2}{*}{$\begin{array}{l}\text { Número de } \\
\text { ítems por } \\
\text { dimensión }\end{array}$} & \multirow[b]{2}{*}{$\begin{array}{l}\text { Alfa de } \\
\text { Cronbach }\end{array}$} & \multicolumn{2}{|c|}{ Consistencia interna } & \multicolumn{2}{|c|}{ Poder discriminante } \\
\hline & & & $\begin{array}{c}\text { Punto- } \\
\text { Dimensión } \\
\text { Rango Rho }\end{array}$ & $\begin{array}{c}\% \\
\text { Exxito }\end{array}$ & $\begin{array}{l}\text { Punto-otra } \\
\text { dimensión } \\
\text { Rango Rho } \\
\end{array}$ & $\begin{array}{c}\% \\
\text { Éxito }\end{array}$ \\
\hline Incapacidad psicológica & 6 & 0,850 & $0,59-0,81$ & 100 & $0,32-0,66$ & 16,6 \\
\hline Incapacidad social & 5 & 0,819 & $0,47-0,73$ & 100 & $0,29-0,58$ & 33,3 \\
\hline En desventaja & 6 & 0,793 & $0,42-0,77$ & 100 & $0,23-0,60$ & 47,2 \\
\hline Total & 49 & 0,951 & & & & \\
\hline
\end{tabular}

Fuente: elaboración propia

De los 49 ítems explorados, se identificaron datos extremos en dos ítems. El primero, en que el participante refería que su prótesis dental no ajustaba bien y que pertenece a la dimensión de limitación funcional; y el segundo en que refería que por su condición bucal no era capaz de trabajar, que pertenece a la dimensión de desventaja. Esto es consistente con la condición clínica de los pacientes debido a que pocos utilizaban prótesis dental y en la clínica es atípico encontrar pacientes que, por su condición bucal, sean incapaces de trabajar. Se decidió no eliminar estos ítems en el análisis por la estandarización de los datos al proceder de una escala tipo Likert con puntuaciones de 0-5, lo cual no afecta de manera sensible las medidas de tendencia central en caso de utilizarse para los 49 ítems.

Se utilizó la prueba de normalidad Shapiro-Francia que presentó valor estadístico de 0,7292 ( $p=0,0001)$, lo que permite rechazar la hipótesis nual, dado que la muestra de la cual se extrajeron los datos no sigue una distribución normal. De igual manera, las medidas de forma asimetría (rango=-0,800;5,139) y curtosis (rango=-1,282;27,923) también mostraron que los datos no siguen una distribución normal.

En la medición de las características de la matriz, se encontró que la matriz de correlaciones era cuadrada y simétrica, tiene inversa y el determinante de la matriz es $1,250033 e^{-13}$. Además, las correlaciones presentes fueron significativas. Al aplicar las pruebas de matrices, se encontró que la prueba estadística KMO al relacionar los coeficientes de correlación, presentó valor 0,9 entre los ítems del instrumento, lo que adicionalmente indica que el tamaño muestral es adecuado para la implementación del AFE. El test de esfericidad de Barttlet=984,64 ( $p=0,001)$, con 48 grados de libertad, permite concluir que la correlación entre las variables es distinta de cero ${ }^{\circ} \mathrm{e}$.

Al aplicar el método de extracción de ejes principales, la mayor proporción de varianza de todas las variables es explicada por el factor 1 con un autovalor extraído de 15,247 y explicando el 31,117\% de la varianza total; mientras que la proporción de varianza de todas las variables explicada por el factor 2 estuvo en 2,428 alcanzando 
a explicar el 4,995\% de la varianza total y conformando una varianza acumulada de $36,072 \%$. Para el resto de los factores la diferencia entre los aportes a la varianza total tuvieron pequeñas diferencias entre ellos (tabla 3). Este modelo explica el 56,8\% de la varianza acumulada de los factores. Es precisamente el valor obtenido por los autovalores lo que redujo los factores incluidos, los autovalores iniciales disminuyeron los factores de 49 a únicamente 12 factores, pero al seleccionar los valores de la suma de extracción de la carga al cuadrado del total de la varianza explicada; sin embargo, se decide utilizar como referencia el modelo de 12 factores por alcanzar la explicación del $56,8 \%$ de la varianza total de los factores, mientras que la varianza total explicada por el modelo de 7 factores explicaba el 48,67\% de la varianza total a pesar de presentar una distribución más equitativa de la varianza de los factores. Cabe resaltar que en el segundo modelo los factores remanentes se establecieron en 7 que es el número de dimensiones que tiene el instrumento OHIP-49 (tabla 3 y tabla 4). Todos los factores obtuvieron cargas factoriales $>0,40$. De los 49 autovalores obtenidos en ambos modelos, todos fueron mayores a 0 , dado que no se encontraron valores negativos queda soportada la decisión de factorizar la matriz.

De acuerdo con el valor de las comunalidades, en el modelo con 12 factores las variables consideradas con explicación insuficiente respecto a la afectación por la condición de sus dientes, boca o prótesis son las pertenecientes a la dimensión MP con los ítems: preocupado por los dientes, y ser consciente de sí mismo; mientras que la dimensión LF obtuvo explicación insuficiente para los ítems: problemas mordiendo, alimentos retenidos, dientes no se ven bien, y aliento deteriorado; por su parte, en la dimensión IF la explicación fue insuficiente para los ítems: no cepillado adecuado, gente malentiende palabras, no poder comer con prótesis; para la dimensión DF la explicación insuficiente estuvo en el ítem: dientes sensibles; por otro lado, en la dimensión ED fue poco explicado el ítem: pérdida financiera; mientras que la dimensión IP tuvo insuficiente explicación para el ítem: interrupción del sueño. La única dimensión que obtuvo suficiente explicación para todos sus ítems fue IS (tabla 3) para un total de 12 factores con explicación insuficiente; esta cantidad se duplica a 23 ítems con explicación insuficiente en el modelo con 7 factores (tabla 4). 
Tabla 3. Análisis Factorial Exploratorio con un modelo de 12 factores, utilizando como referencia autovalores $>1$.

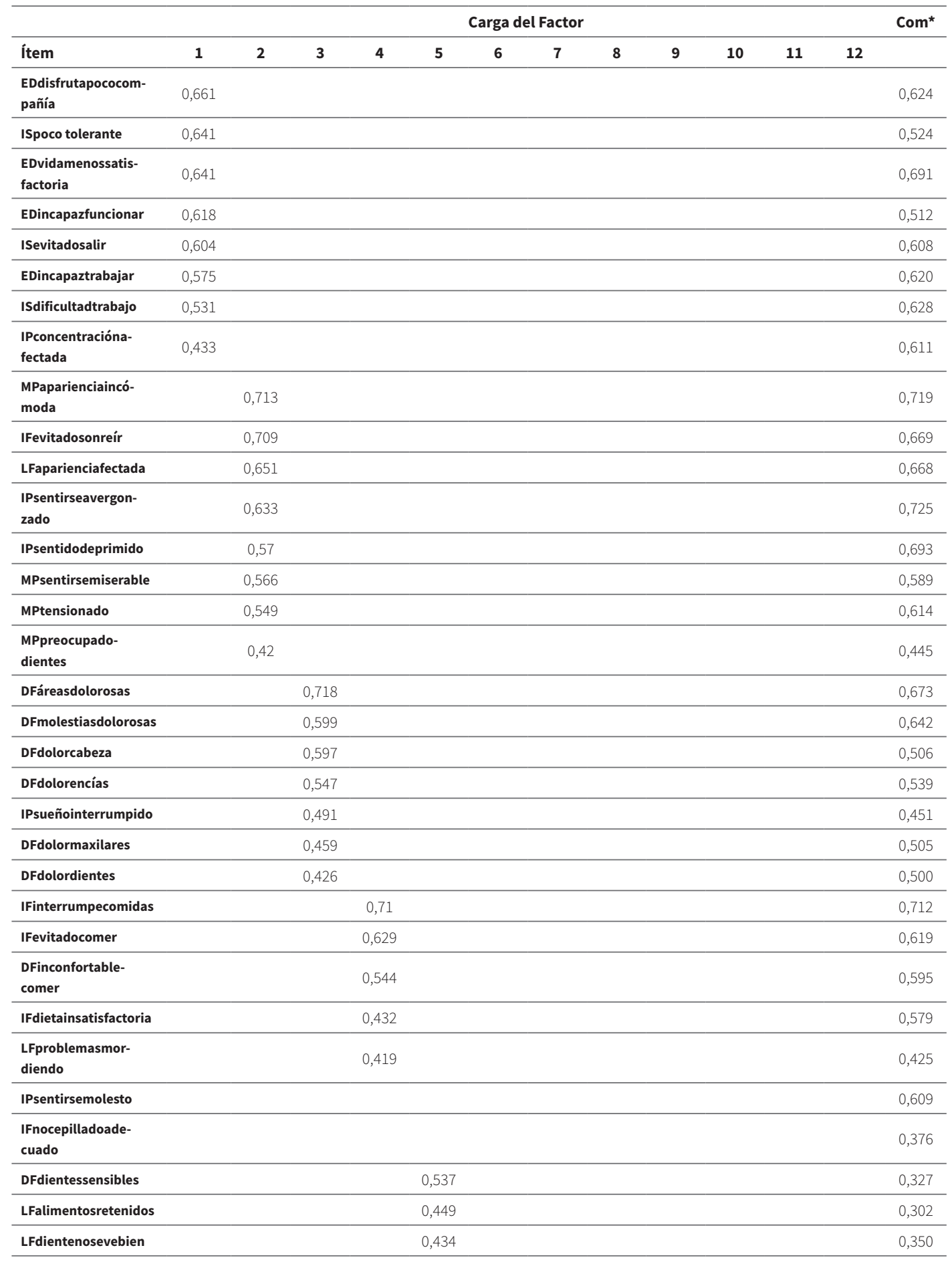


Evaluación de las propiedades psicométricas de reproducibilidad del Oral Health Impact Profile

(OHIP-49) entre pacientes con enfermedad periodontal

(viene)

\begin{tabular}{|c|c|c|c|c|c|c|c|c|c|c|c|c|c|}
\hline \multirow[b]{2}{*}{ Ítem } & \multicolumn{12}{|c|}{ Carga del Factor } & \multirow[t]{2}{*}{ Com } \\
\hline & 1 & 2 & 3 & 4 & 5 & 6 & 7 & 8 & 9 & 10 & 11 & 12 & \\
\hline LFalientodeteriorado & & & & & 0,421 & & & & & & & & 0,470 \\
\hline LFprótesisnoajusta & & & & & & 0,828 & & & & & & & 0,766 \\
\hline DFinconfortable & & & & & & 0,679 & & & & & & & 0,533 \\
\hline IFnocomerconprótesis & & & & & & 0,61 & & & & & & & 0,441 \\
\hline $\begin{array}{l}\text { LFproblemaspronun- } \\
\text { ciando }\end{array}$ & & & & & & & 0,695 & & & & & & 0,629 \\
\hline IFhabla pococlaro & & & & & & & 0,572 & & & & & & 0,684 \\
\hline $\begin{array}{l}\text { IFgentemaltendiente- } \\
\text { palabras }\end{array}$ & & & & & & & 0,52 & & & & & & 0,451 \\
\hline EDpérdidafinanciera & & & & & & & & & & & & & 0,246 \\
\hline LFsaborempeorado & & & & & & & & 0,62 & & & & & 0,560 \\
\hline $\begin{array}{l}\text { IFmenossaborali- } \\
\text { mentos }\end{array}$ & 0,44 & & & & & & & 0,583 & & & & & 0,787 \\
\hline LFempeorada & & & & & & & & & 0,636 & & & & 0,633 \\
\hline $\begin{array}{l}\text { EDsaludgeneralem- } \\
\text { peorada }\end{array}$ & 0,428 & & & & & & & & 0,479 & & & & 0,618 \\
\hline $\begin{array}{l}\text { ISproblemasalrela- } \\
\text { cionarse }\end{array}$ & 0,438 & & & & & & & & & 0,497 & & & 0,732 \\
\hline ISirritadoconotros & & & & & & & & & & 0,471 & & & 0,641 \\
\hline IPdifícilrelajarse & & & & & & & & & & & 0,468 & & 0,722 \\
\hline MPconscientesimismo & & & & & & & & & & & & 0,445 & 0,256 \\
\hline Autovalor & 15,247 & 2,428 & 1,972 & 1,545 & 1,146 & 1,007 & 1,000 & 0,948 & 0,759 & 0,664 & 0,542 & 0,502 & \\
\hline$\%$ Varianza & 31,117 & 4,995 & 4,025 & 3,153 & 2,339 & 2,198 & 2,042 & 1,936 & 1,548 & 1,356 & 1,106 & 1,025 & \\
\hline Varianza aumulada & 31,117 & 36,072 & 40,097 & 43,250 & 45,590 & 47,787 & 49,829 & 51,765 & 53,313 & 54,669 & 55,774 & 56,800 & \\
\hline
\end{tabular}

${ }^{*}$ Comunalidad descrita como varianza de los ítems que se explican con las variables extraídas.

Fuente: elaboración propia.

Tabla 4. Análisis factorial exploratorio con un modelo de 7 factores utilizando como referencia la suma de extracción de la carga al cuadrado del total de la varianza explicada.

\begin{tabular}{|c|c|c|c|c|c|c|c|c|}
\hline \multirow[b]{2}{*}{ Ítem } & \multicolumn{8}{|c|}{ Carga del Factor } \\
\hline & 1 & 2 & 3 & 4 & 5 & 6 & 7 & Com $^{\star}$ \\
\hline EDvidamenossatisfactoria & 0,672 & & & & & & & 0,660 \\
\hline EDdisfrutapococompañía & 0,645 & & & & & & & 0,581 \\
\hline EDincapazfuncionar & 0,635 & & & & & & & 0,461 \\
\hline ISevitadosalir & 0,602 & & & & & & & 0,566 \\
\hline ISpocotolerante & 0,598 & & & & & & & 0,457 \\
\hline EDincapaztrabajar & 0,58 & & & & & & & 0,447 \\
\hline
\end{tabular}


(viene)

\begin{tabular}{|c|c|c|c|c|c|c|c|c|}
\hline & & & & Carga & actor & & & \\
\hline Ítem & 1 & 2 & 3 & 4 & 5 & 6 & 7 & Com* \\
\hline ISdificultadtrabajo & 0,55 & & & & & & & 0,615 \\
\hline IPconcentraciónafectada & 0,507 & & & & & & & 0,579 \\
\hline IPdificilrelajarse & 0,466 & & & & & & & 0,551 \\
\hline ISirritadoconotros & 0,447 & & & & & & & 0,510 \\
\hline IFdietainsatisfactoria & & & & & & & & 0,494 \\
\hline IPsentirsemolesto & & & & & & & & 0,563 \\
\hline EDpérdidafinanciera & & & & & & & & 0,133 \\
\hline DFareasdolorosas & & 0,677 & & & & & & 0,554 \\
\hline DFdolorencias & & 0,63 & & & & & & 0,492 \\
\hline DFmolestiasdolorosas & & 0,603 & & & & & & 0,509 \\
\hline DFdolormaxilares & & 0,583 & & & & & & 0,422 \\
\hline DFdolordientes & & 0,54 & & & & & & 0,478 \\
\hline DFdolorcabeza & & 0,535 & & & & & & 0,382 \\
\hline IPsueñointerrumpido & & 0,43 & & & & & & 0,351 \\
\hline MPpreocupadoxdientes & & 0,427 & & & & & & 0,434 \\
\hline DFdientessensibles & & & & & & & & 0,170 \\
\hline LFdientenosevebien & & & & & & & & 0,266 \\
\hline LFalimentosretenidos & & & & & & & & 0,199 \\
\hline MPaparienciaincomoda & & & 0,693 & & & & & 0,671 \\
\hline IFevitadosonreir & & & 0,681 & & & & & 0,629 \\
\hline IPsentirseavergonzado & & & 0,666 & & & & & 0,698 \\
\hline LFaparienciafectada & & & 0,618 & & & & & 0,635 \\
\hline IPsentidodeprimido & & & 0,55 & & & & & 0,629 \\
\hline MPsentirsemiserable & & & 0,526 & & & & & 0,498 \\
\hline MPtensionado & & & 0,525 & & & & & 0,589 \\
\hline IFinterrumpecomidas & & & & 0,594 & & & & 0,561 \\
\hline DFinconfortablecomer & & & & 0,564 & & & & 0,563 \\
\hline IFevitadocomer & & & & 0,561 & & & & 0,552 \\
\hline LFproblemasmordiendo & & & & 0,489 & & & & 0,402 \\
\hline IFnocepilladoadecuado & & & & & & & & 0,341 \\
\hline MPconscientesimismo & & & & & & & & 0,100 \\
\hline IFhablapococlaro & & & & & 0,586 & & & 0,634 \\
\hline ISproblemasalrelacionarse & & & & & 0,523 & & & 0,586 \\
\hline IFgentemaltendientepalabras & & & & & 0,481 & & & 0,392 \\
\hline IFmenossaboralimentos & & & & & 0,48 & & & 0,627 \\
\hline LFsaborempeorado & & & & & 0,464 & & & 0,464 \\
\hline LFalientodeteriorado & & & & & & & & 0,333 \\
\hline DFprotesisinconfortable & & & & & & 0,738 & & 0,568 \\
\hline LFprótesisnoajusta & & & & & & 0,719 & & 0,588 \\
\hline
\end{tabular}




\begin{tabular}{|c|c|c|c|c|c|c|c|c|}
\hline \multirow[b]{2}{*}{ İtem } & \multicolumn{8}{|c|}{ Carga del Factor } \\
\hline & 1 & 2 & 3 & 4 & 5 & 6 & 7 & Com $^{*}$ \\
\hline IFnocomerconprotesis & & & & & & 0,573 & & 0,370 \\
\hline LFproblemaspronunciando & & & & & & & & 0,432 \\
\hline LFdigectiónempeorada & & & & & & & 0,552 & 0,517 \\
\hline EDsaludgeneralempeorada & 0,431 & & & & & & 0,547 & 0,600 \\
\hline Autovalor & 5,739 & 4,457 & 4,367 & 2,962 & 2,487 & 2,104 & $1,1,736$ & \\
\hline$\%$ Varianza & 11,712 & 9,096 & 8,913 & 6,045 & 5,076 & 4,293 & 3,544 & \\
\hline Varianza aumulada & 11,712 & 20,808 & 29,721 & 35,766 & 40,842 & 45,135 & 48,679 & \\
\hline
\end{tabular}

*Comunalidad descrita como varianza de los ítems que se explican con las variables extraídas.

Fuente: elaboración propia.

En la matriz de residuales, los valores encontrados permiten confirmar que los factores extraídos reproducen adecuadamente la matriz de correlación; de forma complementaria, la presencia de algunos ítems con valores moderados $(0,05 ; 0,10)$ o con residuales grandes $(>0,10)$ sugiere que aún quedan hay factores remanentes por extraer. En la tabla 3 se encontraron 3 ítems que no aportaron a ningún factor, las cuales son: ED pérdida financiera, IF cepillado inadecuado, IP sentirse molesto. Asimismo, en la tabla 4 se observan 10 ítems que no aportan a ningún factor y estos son: LF problemas pronunciando, ED pérdida financiera, IP sentirse molesto, IF dieta insatisfactoria, DF dientes sensibles, LF dientes no se ven bien, LF alimentos retenidos, IF cepillado inadecuado, MP consciente de sí mismo, LF aliento deteriorado.

En el momento de considerar la reducción del instrumento, los ítems que no aportan carga factorial a ningún factor o que presentan comunalidades débiles, deben ser considerados desde la posible relevancia del ítem dentro de la escala y, en el caso de no cumplir con con ninguna de estas características, estos ítems deben ser eliminados y, posteriormente, realizar un nuevo AFE con los ítems remanentes.

El gráfico de sedimentación es consistente con los datos obtenidos para el total de la varianza explicada, donde el primer autovalor el que más aporta a la explicación del primer factor; mostrando con rezago cómo los autovalores siguientes aportan en mucha menor proporción a la explicación de los factores y, por lo tanto, a la justificación de los ítems, donde al utilizar el criterio de Catell se encontró que solo 4 factores remanentes por encima de la línea cuentan con la máxima cantidad de varianza en los 49 factores.

El comportamiento del instrumento presentó la mayor afectación negativa de la CVRSO en las dimensiones LF, DF, y MP; a pesar de esto los puntajes tanto de promedio como de posición se consideran bajos teniendo en cuenta el rango de los valores de respuesta (tabla 5). 
Tabla 5. Descripción de la calidad de vida relacionada con la salud oral por los dominios de la escala OHIP-49.

\begin{tabular}{lccc}
\hline \multicolumn{1}{c}{ Dimensión } & Media $\mathbf{\pm ~ D E}$ & $\begin{array}{c}\text { Mediana (Rango } \\
\text { intercuartílico) }\end{array}$ & Rango de respuesta \\
\hline Limitación funcional & $10,14(6,26)$ & $9(5-15)$ & $0-33$ \\
\hline Dolor físico & $9,15(6,08)$ & $9(4-14)$ & $0-31$ \\
\hline Malestar psicológico & $8,61(4,75)$ & $8(5-12)$ & $0-20$ \\
\hline Incapacidad física & $6,41(6,34)$ & $5(0-10)$ & $0-28$ \\
\hline Incapacidad psicológica & $5,33(5,30)$ & $4(0-9)$ & $0-20$ \\
\hline Incapacidad social & $1,94(3,24)$ & $0(0-3)$ & $0-20$ \\
\hline En desventaja & $2,54(3,85)$ & $0(0-4)$ & $0-18$ \\
\hline Puntaje global & $44,12(29,63)$ & $39(42)$ & $3-145$ \\
\hline
\end{tabular}

Fuente: elaboración propia

En la afectación de la CVRSO según la condición periodontal, los pacientes con periodontitis con estadíos III y IV, por su severidad avanzada, presentaron mayor afectación de la dimensión limitación funcional relacionada con la masticación, deglución, y estética de los dientes. Por otro lado, los participantes con condiciones periodontales menos graves presentaron mayor afectación de la dimensión relacionada con el malestar psicológico que incluye variables que reflejan preocupación, tensión y autopercepción de la apariencia (tabla 6).

Tabla 6. Distribución de las respuestas de pacientes con enfermedad periodontal por cada dimensión de la escala OHIP-49, según el nivel de afectación de la CVRSO.

\begin{tabular}{|c|c|c|c|c|c|c|}
\hline Dimensión & $\begin{array}{c}\text { Efecto } \\
\text { sobre } \\
\text { CVRSO }\end{array}$ & $\begin{array}{c}\text { Gingivitis } \\
\mathbf{n}(\%)\end{array}$ & $\begin{array}{c}\text { P. estadío } \\
\text { III n (\%) }\end{array}$ & $\begin{array}{l}\text { P. estadío } \\
\text { II n (\%) }\end{array}$ & $\begin{array}{l}\text { P. estadío } \\
\text { III-IV n (\%) }\end{array}$ & Total n (\%) \\
\hline \multirow{2}{*}{$\begin{array}{l}\text { Limitación } \\
\text { funcional }\end{array}$} & Mayor ${ }^{\star}$ & $8(3,9)$ & $3(1,4)$ & $3(1,4)$ & $11(5,3)$ & $25(12,1)$ \\
\hline & Menor $^{\star *}$ & $104(50,2)$ & $27(13)$ & $28(13,5)$ & $23(11,1)$ & $182(87,9)$ \\
\hline \multirow{2}{*}{ Dolor físico } & Mayor & $24(11,6)$ & $8(3,9)$ & $4(1,9)$ & $4(1,9)$ & $40(19,3)$ \\
\hline & Menor & $91(44)$ & $27(13)$ & $27(13)$ & $22(10,6)$ & $167(80,7)$ \\
\hline \multirow{2}{*}{$\begin{array}{l}\text { Malestar psico- } \\
\text { lógico }\end{array}$} & Mayor & $43(20,8)$ & $20(9,7)$ & $8(3,9)$ & $14(6,8)$ & $85(41,1)$ \\
\hline & Menor & $72(34,8)$ & $15(7,2)$ & $23(11,1)$ & $12(5,8)$ & $122(58,9)$ \\
\hline \multirow{2}{*}{$\begin{array}{l}\text { Incapacidad } \\
\text { física }\end{array}$} & Mayor & $5(2,4)$ & $3(1,4)$ & $2(1)$ & $3(1,4)$ & $13(6,3)$ \\
\hline & Menor & $110(53,1)$ & $32(15,5)$ & $29(14)$ & $23(11,1)$ & $194(93,7)$ \\
\hline \multirow{2}{*}{$\begin{array}{l}\text { Incapacidad } \\
\text { psicológica }\end{array}$} & Mayor & $7(3,4)$ & $1(0,5)$ & $2(1)$ & $2(1)$ & $12(5,8)$ \\
\hline & Menor & $108(52,2)$ & $34(16,4)$ & $29(14)$ & $24(11,6)$ & $195(94,2)$ \\
\hline
\end{tabular}




\begin{tabular}{lcccccc}
\hline \multicolumn{1}{c}{ Dimensión } & $\begin{array}{c}\text { Efecto } \\
\text { sobre } \\
\text { CVRSO }\end{array}$ & $\begin{array}{c}\text { Gingivitis } \\
\mathbf{n}(\mathbf{\%})\end{array}$ & $\begin{array}{c}\text { P. estadío } \\
\text { III } \mathbf{n}(\%)\end{array}$ & $\begin{array}{c}\text { P. estadío } \\
\text { II } \mathbf{n}(\%)\end{array}$ & $\begin{array}{c}\text { P. estadío } \\
\text { III-IV n (\%) }\end{array}$ & Total n (\%) \\
\hline $\begin{array}{l}\text { Incapacidad } \\
\text { social }\end{array}$ & Mayor & $1(0,5)$ & $2(1)$ & $1(0,5)$ & $4(1,9)$ & $8(3,9)$ \\
\cline { 2 - 7 } En desventaja & Menor & $114(55,1)$ & $33(15,9)$ & $30(14,5)$ & $22(10,6)$ & $199(96,1)$ \\
\cline { 2 - 7 } & Mayor & $3(1,4)$ & $3(1,4)$ & $2(1)$ & $4(1,9)$ & $12(5,8)$ \\
\hline
\end{tabular}

*Agrupación de participantes con el efecto más bajo sobre la CVRSO (0-2).

“Agrupación de participantes con el efecto más alto sobre la CVRSO (3-4).

Fuente: elaboración propia

\section{Discusión}

Artículos tanto de estudios observacionales como experimentales, no reportan los métodos y resultados completos de la verificación de las propiedades psicométricas de los instrumentos (35), lo que afecta la validez interna de los resultados a consideración, pues no es posible verificar el comportamiento del instrumento en la población y verificar que realmente mida el constructo propuesto. Algunos autores consideran que el OHIP-14 tiene un efecto piso (36) (37), por esta razón, en este estudio se consideró el OHIP-49 como la opción idónea para controlar el efecto piso al ofrecer mayor variabilidad en las opciones de respuesta, y quizás ser más sensible a las manifestaciones de la enfermedad periodontal, con la limitante de que no se encuentran muchos estudios que hagan una evaluación psicométrica completa de este instrumento, a pesar de que se usa en distintos países.

Un grupo de investigadores evaluó, a través del análisis factorial confirmatorio, la validez de constructo del OHIP-49 en países como Croacia, Alemania, Hungría, Japón, Eslovenia y Suiza; dentro de sus hallazgos resaltan la multidimensionalidad del modelo; aun con la disminución de las siete dimensiones aún se puede mantener la estructura latente del modelo. Los investigadores encontraron en su análisis la evidencia de un fuerte factor general que subyace a los ítems que conforman el OHIP-49, lo que le permite mantenerse estable en su estructura a través de las culturas y las poblaciones en que es implementado el instrumento (38). Esto coincide con los hallazgos de este estudio debido a que una gran proporción de la varianza total acumulada puede ser explicada por menos factores, a pesar de no estar incluido en los resultados al implementar el método de rotación QUARTIMAX con normalización de Kaiser en la 
extracción realizada con el análisis de ejes principales. En este método a través de la simplificación de sus filas y, teniendo en cuenta que incluye un análisis menos robusto, se encontró que 38 de los 49 ítems tenían sus mayores cargas aportantes al primer factor, lo cual es consistente con lo descrito por Santos et al (39), acerca de tendencia a la unidimensionalidad de la escala desde su versión extendida hasta la resumida. Al pensar en seleccionar el mejor método de extracción, también se aplicó el análisis de componentes principales, pero las cargas de los componentes de la matriz estuvieron muy por encima de la carga de los factores utilizando el método de ejes principales. Esto puede deberse a que el análisis de componentes principales no discrimina entre la varianza común y la específica por lo cual tiende a inflar los valores (32), por esa razón, sumado a no ser un método de análisis del factor, sino de reducción de datos, fue excluido de este estudio.

En esta investigación no se aplicó el Análisis Factorial Confirmatorio (AFC) porque, desde hace algunos años, en la literatura se describen sus limitaciones, pues requiere confirmar estructuras factoriales debido a su alto nivel de restricción. Para cubrir esta necesidad, la sugerencia es utilizar el Modelo de Ecuaciones Estructurales Exploratorio, como híbrido entre AFE y AFC, que se caracteriza por aplicar las fortalezas de ambos (40)-(42).

Hasta el momento, en la literatura no se han encontrado reportes del uso del OHIP-49 en pacientes con enfermedad periodontal, se espera que esta investigación sea un referente para la aplicación y análisis de este instrumento. Un estudio encontró, al aplicar el OHIP-14, que en una población de 207 participantes con rango de edad entre 18 y 82 años, el instrumento obtuvo un de Cronbach=0,95 (rango entre dimensiones=0,74;0.85), este rango es mayor (64 años) a los rangos de edad incluidos en estudios de evaluación del instrumento por otros autores. En estudios con 122 participantes con edades superiores a 60 años residentes en Adelaide, el $\alpha$ de Cronbach por dimensión estuvo entre el rango 0,70 a 0,83. Otra investigación realizada en Ontario, Canadá, con participantes mayores de 50 años reportó entre sus dimensiones un $\alpha$ de Cronbach entre 0,80 y 0,90. En otro estudio con 1642 participantes adultos mayores realizado en Carolina del Norte, Estados Unidos, el coeficiente de $\alpha$ de Cronbach para cada una de las dimensiones del instrumento fue de mínimo 0,96 para cada dimensión (43), estos altos valores en el $\alpha$ de Cronbach sugiere la presencia de duplicidad entre los ítems.

La presencia de duplicidad entre los ítems del instrumento es consistente con lo referido por el creador del OHIP-49, dando soporte a la reducción del instrumento en su versión corta denominada OHIP-14. Para la extracción de los datos, el autor tuvo en cuenta los autovalores y las cargas factoriales, utilizando el método de componentes 
principales complementado con la regresión de mínimos cuadrados asumiendo la independencia entre los datos. A medida que extraía ítems medía el coeficiente de $\alpha$ de Cronbach global y por dimensión, con 39 ítems el de Cronbach fue de 0,94; al momento de retener 14 ítems durante la extracción de los factores, el $\alpha$ de Cronbach fue de 0,88 (10).

En cuanto a las condiciones clínicas de los pacientes, estudios transversales revelan que las dimensiones de limitación funcional, malestar, y en desventaja social, se ven afectadas de forma consistente entre distintas poblaciones; algo similar, se encontró en pacientes edéntulos del sur de Australia con altos niveles de afectación en las dimensiones limitación funcional e incapacidad física, al compararlos con pacientes dentados; mientras que otras dimensiones no presentaron mayores diferencias entre ellas (43).

La evaluación de las propiedades de reproducibilidad al aplicar el instrumento en pacientes con periodontitis, evidenció la ausencia de rigurosidad en los procesos de evaluación psicométrica en esta población. Debido a que el OHIP es utilizado ampliamente, se espera encontrar publicaciones con la validación transcultural del instrumento en apariencia, constructo, contenido y, a través de las propiedades de reproducibilidad. Hasta el momento, solo un artículo evaluó las propiedades correspondientes a reproducibilidad en pacientes con periodontitis, pero no tuvo en cuenta la evaluación de las propiedades de validez del instrumento. Este es el artículo de Al Habashneh et al (22). Los autores describen cómo es su diseño, de corte transversal, con una muestra de 400 participantes conformada por 164 hombres y 236 mujeres, aunque no utilizan el OHIP-49, sino la versión árabe del OHIP-14 obtuvo una fiabilidad de 0,89 representada por el alfa de Cronbach, presentó en coeficiente de correlación de Pearson una consistencia interna con rango 0,49;0,90; y buen poder discriminante en las dimensiones de limitación funcional $(0,13 ; 0,24)$ y malestar psicológico $(0,05 ; 0,10)$, mientras que en las dimensiones dolor físico, discapacidad física y psicológica, discapacidad social y minusvalía, su poder discriminante fue menor. En la prueba test retest la correlación general de la prueba fue de 0,93.

El estudio de Al Habanesh es una buena referencia de lo que se encuentra disponible en la literatura en lo que concerne a la medición de la CVRSO en pacientes con enfermedades periodontales, es una situación frecuente que no permite al lector discernir en cuanto al análisis de las propiedades psicométricas tanto de validez como de reproducibilidad, de manera, que permitan seleccionar un instrumento sobre otro por su excelente comportamiento en la población de estudio y no solo por la frecuencia de su uso. Es necesario considerar que esta es una buena oportunidad para justificar la necesidad de representar las propiedades psicométricas de validez y 
reproducibilidad cada vez que se utilice un instrumento y plantearlo como una primera fase en investigación de validación, dejando como segunda fase el análisis de la validez de contenido junto con todas las propiedades relacionadas con la reproducibilidad de estos instrumentos.

Una limitante de este estudio fue la imposibilidad de utilizar los datos obtenidos en las evaluaciones test-retest, fiablidad inter e intraobservador, debido a la variablidad de los resultados obtenidos en la segunda medición con respecto a la primera, lo cual, se pronosticó por la exposición de los participantes a tratamientos odontológicos posteriores a la primera medición de CVRSO, dado que los investigadores no debían intervenir con el proceso establecido en el cronograma de actividades de las IPS en lo referente a la atención de sus pacientes. Por esta razón, los datos de la segunda medición fueron excluidos del análisis por deberse la variablidad no al instrumento, sino al tratamiento recibido. Por otro lado, los participantes describieron problemas en la comprensión de las preguntas relacionadas con: ser consciente de sí mismo, ser totalmente incapaz de funcionar, encontrar inconfortable comer algún alimento, y sueño que ha sido interrumpido, este último porque en ocasiones lo consideran la interrupción de sus expectativas de vida y no la condición de soñar mientras se duerme. Por lo anterior, para la implementación de la segunda fase de la prueba como adaptación cultural se adicionará la interpretación de la pregunta. Se debe aclarar que los resultados presentados relacionados con la CVRSO son una aproximación, porque antes de medir el constructo en la segunda fase de esta investigación, utilizando el tamaño muestral calculado para nuestro estudio de cohorte, el primer paso es verificar que el instrumento mida adecuadamente el constructo en la población de interés y esos son los resultados que se presentan aquí.

La evaluación de las propiedades psicométricas de los instrumentos se vuelve relevante debido al incremento del desarrollo de estudios internacionales al igual que de la diversidad cultural dentro de un mismo país. Esto requiere contar con instrumentos de evaluación que estén adaptados culturalmente en los distintos países. Por esta razón, la oms recomienda desarrollar e implementar indicadores de la salud que estén estandarizados, promoviendo a la vez la colaboración a través de investigaciones multicéntricas preferiblemente con alcance internacional y conseguir instrumentos de medición en el área de la salud que estén validados con la finalidad de desarrollar estudios comparativos a nivel internacional (44). 


\section{Conclusiones}

En el área de la salud, no se cuenta hasta el momento con una guía estandarizada para evaluar las propiedades psicométricas de los instrumentos de medición. Los investigadores tienen la necesidad de utilizar herramientas del campo de la educación o la psicología y los artículos tanto de estudios observacionales como experimentales, no reportan los métodos y resultados completos de la verificación, aclarando que el proceso puede ser modificado según el objetivo y las características del instrumento que se aplique (44) (45). Estos procedimientos deben ser complementados con el entendimiento estadístico de las bases para la selección de los métodos implementados en la validación de instrumentos de medición. Por eso, este documento espera ser una guía con el soporte estadístico que permita a los odontólogos explorar y desarrollar productos en un área de continuo crecimiento para la profesión.

El OHIP-49, en esta prueba piloto, presentó buen comportamiento en lo referente a las propiedades psicométricas de reproducibilidad, pero al momento de revisar los porcentajes de éxito, específicamente en el poder discriminante, generó dudas con respecto a la estructura del instrumento, lo que terminó en la necesidad práctica de implementar el AFE, mostrando que así como hay ítems que no aportan a sus dimensiones, de forma similar hay ítems que aportan a distintas dimensiones, lo que es consistente con el valor global del alpha de Cronbach al evidenciar duplicidad. Es necesario, analizar si las propiedades psicométricas de reproducibilidad pueden mostrar la validez de contenido e incluso de constructo del instrumento; más aún, en escalas de uso ampliamente extendido como el OHIP-49 (46), llama la atención la aparente ausencia de literatura que de forma secuencial evalúe tanto las propiedades de validez como de reproducibilidad del instrumento. De igual manera, es necesario contrastar los resultados obtenidos en la evaluación de la reproducibilidad del OHIP49 con las encontradas en el OHIP-14 porque debido a la tendencia en los valores de consistencia interna y porcentajes de éxito, es probable que se obtengan resultados similares con el uso de la versión corta del instrumento.

En los estadíos avanzados de la periodontitis, su manejo clínico y quirúrgico lo realiza el especialista en periodoncia, enfocado en prevenir la progresión de la enfermedad; pero en Colombia este tratamiento no está cubierto por el Plan de Beneficios en Salud, lo que obliga al paciente, en ocasiones, a interponer acciones legales para acceder al tratamiento. Es necesario que el odontólogo se familiarice con el diseño, implementación y análisis de escalas de medición en salud y que desde su experticia pueda complementar los datos obtenidos de estudios poblacionales, de 
intervención y costos; de manera que logre articularlos con el desarrollo de políticas públicas con base en una perspectiva holística para la búsqueda de solución ante la problemática de los pacientes.

\section{Conflicto de intereses}

Los investigadores declaran que no presentan ningún conflicto de interés que pueda influir sobre los resultados obtenidos de esta investigación.

\section{Referencias}

1. Gift HC, Atchison KA, Dayton CM. Conceptualizing Oral Health and Oral Health-Related Quality of Life. Soc Sci Med. 1997; 44(5): 601-608. https://doi.org/10.1016/S0277-9536(96)00211-0

2. Araújo AC da S, Gusmão ES, Batista JEM, Cimões R. Impact of Periodontal Disease on Quality of Life. Quintessence Int. 2010; 41(6): e111-118.

3. Williams RC. GRJ. Enfermedad periodontal y salud general: una guía para el clínico. 1ra edición. Pennsylvania, USA: Professional Audience Communications, Inc; 2011.

4. Pihlstrom BL, Michalowicz BS, Johnson NW. Periodontal Diseases. Lancet. 2005; 366(9499): 1809-1820. https://doi.org/10.1016/S0140-6736(05)67728-8

5. Durham J, Fraser HM, McCracken GI, Stone KM, John MT, Preshaw PM. Impact of Periodontitis on Oral Health-Related Quality of Life. J Dent. 2013; 41(4): 370-376. https://doi.org/10.1016/j. jdent.2013.01.008

6. O'Dowd LK, Durham J, McCracken GI, Preshaw PM. Patients' Experiences of the Impact of Periodontal Disease. J Clin Periodontol. 2010; 37(4): 334-339. https://doi. org/10.1111/j.1600-051X.2010.01545.x

7. Kassebaum NJ, Bernabé E, Dahiya M, Bhandari B, Murray CJL, Marcenes W. Global Burden of Severe Periodontitis in 1990-2010: A Systematic Review and Meta-Regression. J Dent Res. 2014; 93(11): 1045-1053. https://doi.org/10.1177/0022034514552491

8. República de Colombia. Ministerio de salud y protección social. IV Estudio nacional de salud bucal ENSAB IV 2013-2014. 2014 Disponible en: https://www.minsalud.gov.co/sites/rid/Lists/ BibliotecaDigital/RIDE/VS/PP/ENSAB-IV-Situacion-Bucal-Actual.pdf 
9. Slade GD, Spencer AJ. Development and Evaluation of the Oral Health Impact Profile. Community Dent Health. 1994;11(1): 3-11.

10. Slade GD. Derivation and Validation of a Short-Form Oral Health Impact Profile. Community Dent Oral Epidemiol. 1997; 25(4):284-290.

11. Wong MCM, Lo ECM, McMillan AS. Validation of a Chinese Version of the Oral Health Impact Profile (OHIP). Community Dent Oral Epidemiol. 2002; 30(6): 423-430.

12. Grecu A-G, Dudea D, Balazsi R, Dumitrascu DL. Romanian Version of the Oral Health Impact Profile-49 Questionnaire: Validation and Preliminary Assessment of the Psychometrical Properties. Clujul Med. 2015; 88(4): 530-536. https://doi.org/10.15386/cjmed-551

13. Deshpande NC, Nawathe AA. Translation and Validation of Hindi Version of Oral Health Impact Profile-14. J Indian Soc Periodontol. 2015; 19(2): 208-210. https://doi. org/10.4103/0972-124X.145806

14. León S, Bravo-Cavicchioli D, Correa-Beltrán G, Giacaman RA. Validation of the Spanish Version of the Oral Health Impact Profile (OHIP-14Sp) in Elderly Chileans. BMC Oral Health. 2014; 14: 95. https://doi.org/10.1186/1472-6831-14-95

15. Castrejón-Pérez RC, Borges-Yáñez SA. Derivation of the Short Form of the Oral Health Impact Profile in Spanish (OHIP-EE-14). Gerodontology. 2012;29(2):155-158. https://doi. $\operatorname{org} / 10.1111 / j .1741-2358.2012 .00613 . x$

16. Castrejón-Pérez RC, Borges-Yáñez SA, Irigoyen-Camacho ME. Validation of an Instrument for Measuring the Effects of Oral Health on the Quality of Life of Older Adults in Mexico. Rev Panam Salud Pública. 2010; 27(5): 321-329.

17. de Souza RF, Terada ASSD, Vecchia MPD, Regis RR, Zanini AP, Compagnoni MA. Validation of the Brazilian Versions of two inventories for measuring Oral Health-Related Quality of Life of Edentulous Subjects. Gerodontology. 2012; 29(2): e88-95. https://doi. $\operatorname{org} / 10.1111 / j .1741-2358.2010 .00417 . x$

18. Liu JY, Pow EHN, Chen ZF, Zheng J, ZhangXC, Chen J. The Mandarin Chinese Shortened Version ofOral Health ImpactProfileforPartially EdentatePatientswith Implant-Supported Prostheses. J Oral Rehabil. 2012; 39(8): 591-599. https://doi.org/10.1111/j.1365-2842.2012.02292.x

19. Papagiannopoulou V, Oulis CJ, Papaioannou W, Antonogeorgos G, Yfantopoulos J. Validation of a Greek Version of the Oral Health Impact Profile (OHIP-14) for Use Among Adults. Health Qual Life Outcomes. 2012;10:7. https://doi.org/10.1186/1477-7525-10-7 
20. Meusel DRDZ, Ramacciato JC, Motta RHL, Brito Júnior RB, Flório FM. Impact of the Severity of Chronic Periodontal Disease on Quality of Life. J Oral Sci. 2015; 57(2): 87-94. https://doi. org/10.2334/josnusd.57.87

21. Bernabé E, Marcenes W. Periodontal Disease and Quality of Life in British Adults. J Clin Periodontol. 2010; 37(11): 968-972. https://doi.org/10.1111/j.1600-051X.2010.01627.x

22. Al Habashneh R, Khader YS, Salameh S. Use of the Arabic Version of Oral Health Impact Profile-14 to Evaluate the Impact of Periodontal Disease on Oral Health-Related Quality of Life Among Jordanian Adults. J Oral Sci. 2012; 54(1): 113-120.

23. Al-Harthi LS, Cullinan MP, Leichter JW, Thomson WM. The Impact of Periodontitis on Oral Health-Related Quality of Life: A Review of the Evidence from Observational Studies. Aust Dent J. 2013; 58(3): 274-277; quiz 384. https://doi.org/10.1111/adj.12076

24. Cerón-Bastidas XA. Calidad de vida y su relación con la salud oral en personas de la tercera edad. Rev Nac odontol. 2014; 10(19): 83-90.

25. International Test Commission. The ITC Guidelines for Translating and Adapting Tests (Second edition). 2017. Disponible en: https://www.intestcom.org/files/guideline_test_ adaptation_2ed.pdf

26. Agudelo-Suárez A. D-DV. Calidad de vida relacionada con la salud bucal en adultos mayores que consultan a la IPS Universitaria de Medellín y sus factores asociados. Rev CES Odont. 2013; 26(1): 10-23.

27. Cardona-Arias J.A. L-TJA. Construcción y validación de escalas de medición en salud: revisión de propiedades psicométricas. Archivos de Medicina. 2015; 11(3:1): 1-10.

28. López R, Baelum V. Spanish Version of the Oral Health Impact Profile (OHIP-Sp). BMC Oral Health. 2006; 6:11.

29. Martin RC. Diseño y validación de cuestionarios. Matronas Profesión. 2004; 5(17): 23-29.

30. Campo-Arias A OH. Propiedades psicométricas de una escala: la consistencia interna. Rev salud pública. 2008; 10(5): 831-839.

31. Pett M, Sullivan J, Lackey N. Making Sense of Factor Analysis. Primera edición. Thousand Oaks: Sage Publications; 2003. 
Evaluación de las propiedades psicométricas de reproducibilidad del Oral Health Impact Profile (OHIP-49) entre pacientes con enfermedad periodontal

32. Medrano L, Pérez E. Análisis factorial exploratorio: bases conceptuales y metodológicas. Revista Argentinas de Ciencias del Comportamiento. 2010; 2(1): 58-66.

33. Songwon Seo. A Review and Comparison of Methods for Detecting Outliers in Univariate Data Sets. University of Pittsburgh; 2006. Disponible en: http://d-scholarship.pitt.edu/7948/ 1/Seo.pdf

34. Hair J, Black W, Babin B, Anderson R. Multivariate Data Analysis. 7a edición. Inglaterra: Pearson Custom Library; 2015.

35. Naito M, Yuasa H, Nomura Y, Nakayama T, Hamajima N, Hanada N. Oral Health Status and Health-Related Quality of Life: A Systematic Review. J Oral Sci. 2006; 48(1): 1-7.

36. Jansson H, Wahlin Å, Johansson V, Åkerman S, Lundegren N, Isberg P-E, et al. Impact of Periodontal Disease Experience on Oral Health-Related Quality of Life. J Periodontol. 2014; 85(3): 438-445. doi: 10.1902/jop.2013.130188

37. Wong RMS, Ng SKS, Corbet EF, Keung Leung W. Non-Surgical Periodontal Therapy Improves Oral Health-Related Quality of Life. J Clin Periodontol. 2012; 39(1): 53-61. https://doi. org/10.1111/j.1600-051X.2011.01797.x

38. John MT, Reissmann DR, Feuerstahler L, Waller N, Baba K, Larsson P, et al. Exploratory Factor Analysis of the Oral Health Impact Profile. Journal of Oral Rehabilitation. 2014; 41(9): 635-643. https://doi.org/10.1111/joor.12192

39. Santos CM dos, Oliveira BH de, Nadanovsky P, Hilgert JB, Celeste RK, Hugo FN. The Oral Health Impact Profile-14: A Unidimensional Scale? Cad Saude Publica. 2013; 29(4): 749-757.

40. Lloret-Segura S, Ferreres-Traver A, Hernández-Baeza A, Tomás-Marco I. El análisis factorial exploratorio de los ítems: una guía práctica, revisada y actualizada. Anales de Psicología. 2014; 30(3). https://doi.org/10.6018/analesps.30.3.199361

41. Ferrando J, Anguiano-Carrasco C. El análisis factorial como técnica de investigación en Psicología. Papeles del psicólogo. 2010; 31(1): 18-33.

42. Marsh HW, Lüdtke O, Muthén B, Asparouhov T, Morin AJS, Trautwein U, et al. A New Look at the Big Five Factor Structure Through Exploratory Structural Equation Modeling. Psychological Assessment. 2010; 22(3): 471-491. https://doi.org/10.1037/a0019227

43. Slade GD. The Oral Health Impact Profile. 1ra ed. Chapel Hill: University of North Carolina: Department of Dental Ecology; 1997. 
44. Carvajal A. SRA. ¿Cómo validar un instrumento de medida de la salud? An Sist Sanit Navar. 2011; 34(1): 63-72.

45. Anastasi A. Evolving Concepts of Test Validation. Annual Review of Psychology. 1986; 37(1):1-16.

46. De La Hoz RP, Segura-Cardona A. Instrumentos para medir la calidad de vida relacionada con la salud oral: una revisión sistemática. Salud Uninorte. 2017; 33(3): 504-516. 